\title{
Applying SIFT Descriptors to Stellar Image Matching*
}

\author{
Javier Ruiz-del-Solar, Patricio Loncomilla, and Pablo Zorzi \\ Department of Electrical Engineering, Universidad de Chile
}

\begin{abstract}
Stellar image matching allows to verify if a given pair of images belongs to the same stellar object/area, or knowing that they correspond to the same sky area, to verify if there are some changes between them due to an stellar event (supernova event, changes in the object position, etc). Some applications are stellar photometry, telescope tracking and pointing, robot telescopes, and sky monitoring. However, the matching of stellar images is a hard problem because normally the images are taken using different telescopes, image sensors and settings, as well as from different places, which produces variability in the image's resolution, orientation, and field of view. In this context, the aim of this paper is to propose a robust SIFT-based wide baseline matching technique for stellar images. The proposed technique was evaluated in a new database composed by 100 pairs of galaxies, nebulas and star clusters images, achieving a true positive rate of $87 \%$ with a false positive rate of $1.7 \%$.
\end{abstract}

Keywords: Matching techniques, SIFT descriptors, Stellar Image Matching.

\section{Introduction}

The exponential growth of data information due to large digital sky surveys that astronomy have experienced during the last decades, and the availability of powerful computer capacity have lead to the development of newer, faster and more complex algorithms to handle these enormous amounts of data. There are several types of models and techniques developed in astronomical image processing for different kind of applications such as image enhancement, data reduction, image classification, correlation calculations and pattern recognition, just to name some.

Stellar image matching allows to verify if a given pair of images belongs to the same stellar object/area, or knowing that they correspond to the same sky area, to verify if there are some changes between them due to an stellar event (supernova event, changes in the object position, etc). This matching operation could easily be implemented using the correlation process, but then it is required that the two images have the same size, resolution orientation and view angle, an impossible task to be done even if the images were taken by the same telescope. A more suitable technique to be used in this application is wide baseline matching ${ }^{1}$ using local interest points and descriptors (see for example [2][6][10]). One of the most popular wide baseline

\footnotetext{
* This research was funded by Millennium Nucleus Center for Web Research, Grant P04-067-F, Chile.

${ }^{1}$ In a wide baseline matching operation, images are allowed to be taken from widely separated viewpoints, so that a point in one image may have moved anywhere in the other image.
} 
matching techniques is the so-called SIFT technique, whose original version was proposed by David Lowe in [1], and improved later on in [2]. The principal characteristics of the SIFT descriptors are their invariance to image translations, scaling and rotations. They are also robust to affine transformation, changes in illumination, and minor deformations. Among other applications, besides object recognition in 2D images, the SIFT-based technique has been applied in 3D reconstruction [3], robot localization [4], and in motion tracking and segmentation [5].

In this context, the aim of this work is to match stellar images using a robust SIFTbased wide baseline matching technique. This article is structured as follows. First, a description of the proposed stellar image matching approach is given in section 2 . In section 3 the final results of the applied approach are presented. Finally, section 4 deals with some preliminary conclusions of this work

\section{The Proposed Stellar Image Matching Approach}

The proposed approach uses local descriptors from stellar images as the main information bricks. A local descriptor is a vector of characteristics that describes the content of a squared area that was selected in the image by an interest point detector. Interest point detectors select some interest regions in the image that maximize some local texture criteria, being the interest point the center of an interest region. Thus, if the image is transformed (rotated, resized or translated), the interest points that are detected transform in the same way as the image. Then, as the interest regions are fixed to the content of the image, their characterization (the local descriptors) are invariant to some image transformations, and by matching similar local descriptors from a pair of images, similar structures in both images could be detected.

Several systems to calculate descriptors and match them have been proposed [6][7][8][9] in the last years, achieving impressive results. The system selected in this work to calculate and match local descriptors is based on Lowe's SIFT algorithm [2], because of their excellent trade-off between precision, speed and low number of false positives. The system selects interest points by blurring an image several times with increasing strength and stacking them in a pyramid. By subtracting consecutive pairs of images from the pyramid, a difference pyramid is generated. Points that are maxima or minima in the difference pyramid are selected as interest points, with a defined position (position of the point in the image), scale (level of the point in the difference pyramid) and orientation (main gradient direction in a zone around the point). The position and scale of the points is improved by interpolating data in the pyramid. Each detected interest point is characterized by a SIFT descriptor, that is a histogram of the orientations of the gradient around the interest point. By matching similar interest points between a pair of images, tentative matches of corresponding points can be obtained. Similar structures in both images are found by finding coherent subgroups of matches that map one image into the other one. For detecting these coherent groups of matches, a similarity transformation (rotation, translation and resizing) is considered, and their possible parameters are considered as a parameters space using a Hough transform approach. The parameters space is quantized in bins, then each match votes for compatible bins in the parameter space (vote for the parameters of the transformations that are able to transform one positionorientation-scale point into the other). The probability that a bin correspond to a real 
detected structure is evaluated by considering the probability that a random wrong match vote for the bin, the amount of votes contained in the bin and the total number of tentative matches. For a more detailed explanation, refer to [2], [7] and [10].

As distinct small regions in stellar images are nearly similar, almost all the tentative matches are wrong, and the described approach is not powerful enough to solve the problem, because the discriminative power of the Hough space probability test is not strong enough. Then, additional verification tests are needed to discard false detections. These extra verification stages are included in the L\&R system [11][12][13], which has demonstrated a superior performance than the original Lowe's algorithm [10].

The L\&R system considers four main stages: (i) Generation of local interest points, (ii) Computation of the SIFT descriptors, (iii) SIFT-matching using nearest descriptors, and (iv) Transformation Computation and Hypothesis Rejection Tests. The first three stages are the standard stages proposed by Lowe and already described, while the fourth stage is used to reduce the number of false matches, giving robustness to the whole system. This stage is implemented by the following procedure:

1. Similarity transformations are determined using the Hough transform (see description in [7][10]). After the Hough transform is computed a set of bins, each one corresponding to a similarity transformation is determined. Then:

a. Invalid bins (those that have less than 4 votes) are eliminated.

b. Q is defined as the set of all valid candidate bins (all those not eliminated in 1.a).

c. $\mathrm{R}$ is defined as the set of all accepted bins. This set is initialized as a void set.

2. For each bin $B$ in $Q$ applied the following tests (the procedure is optimized for obtaining high processing speed by applying less time consuming tests first):

a. If the bin has a more voted direct neighbour in the Hough space, delete bin $B$ from $\mathrm{Q}$ and go to 2 .

b. Calculate the linear correlation $\mathrm{r}_{\mathrm{REF}}$ of the interest points of the matches of $\mathrm{B}$ that lie in the reference image, and the linear correlation $r_{\mathrm{TEST}}$ for the points that lie in the test image. If the absolute value of any of these two coefficients is high, delete bin B from Q and go to 2 .

c. Calculate the fast probability $\mathrm{P}_{\mathrm{FAST}}$ (probability of the Bin) coefficient to $\mathrm{B}$. If $\mathrm{P}_{\mathrm{FAST}}$ is lower than $\mathrm{P}_{\mathrm{TH} 1}$ (probability of the voted Bin being right), delete bin $\mathrm{B}$ from $\mathrm{Q}$ and go to 2 . This procedure is described in [10].

d. Calculate an initial affine transform $T_{B}$ for $B$.

e. If $\mathrm{T}_{\mathrm{B}}$ has a strong affine distortion, delete bin $\mathrm{B}$ from $\mathrm{Q}$ and go to 2. This geometrical distortion verification stage is described in [11].

f. Top down matching: Matches from all the bins in $\mathrm{Q}$ who are compatible with the affine transform $T_{B}$ are cloned and added to bin $B$. Duplication of matches inside $\mathrm{B}$ is avoided.

g. Calculate Lowe's probability of bin B. If it is lower than $\mathrm{P}_{\mathrm{TH} 2}$ (probability of the voted Bin being right), delete bin B from Q and go to 2. See [7].

h. Apply RANSAC for finding a more precise transformation. In case that RANSAC success, a new $\mathrm{T}_{\mathrm{B}}$ transformation is calculated.

i. Accept $B$ and $T_{B}$, i.e., exclude $B$ from $Q$ and include it in $R$. 
3. For all pairs $\left(B_{i}, B_{j}\right)$ in $R$, check it they may be fused into a new bin $B_{k}$. If the bins may be fused and one of them is RANSAC-approved, do not fuse them and delete the other in order to preserve exactitude. If the two bins are RANSAC-approved, delete the least probable. Repeat this until all the possible pairs (including the new created bins) have been checked. This fusion procedure is described in [11].

4. For any bin B in R, apply semi-local constraints procedure to all the matches in B. The matches from $\mathrm{B}$ who are incompatible with the constraints are deleted. If any match is deleted, $T_{B}$ is recalculated. This procedure is described in [14].

5. For any bin $B$ in $R$, calculate the pixel correlation $r_{P I X E L}$ using $T_{B}$. If $r_{P I X E L}$ is low, delete $\mathrm{B}$ from $\mathrm{R}$. This correlation test is described in [11].

6. Assign a priority to all the bins (transformations) in R. A more probable bin (in the Lowe's probability sense) has better priority than a less probable one, but any RANSAC-approved bin has better priority than any non RANSAC-approved one.

\section{Stellar Image Matching Results}

To test the proposed stellar image matching approach, an image database consisting of 100 pairs of reference-test stellar images $\left\{\left(I_{2 k-1}, I_{2 k}\right), k=1 . .100\right\}$ was built (see examples in figs. 2-4). To achieve good statistical results, the image database considers three different types of stellar images: 32 pair of galaxies, 37 pair of nebulas and 31 pair of star clusters. Each reference image shows a different, single stellar object. The same object appears in the corresponding test image, which can contains variations in position and view angle, as well as size (number of pixels), resolution and camera parameters. The selected images have to fulfill two main conditions: first, each reference image must have a higher resolution than its test counterpart, and secondly, a pair of reference-test images must not belong to the same observatory. All stellar images were extracted from public databases.

Matching experiments were carried out in all 100x100 image pairs, i.e. by matching every test image to every reference image. Each matching experiment generates a variable number of affine transformations ( 0 to 10 , or even more in some cases), although just one should be obtained when the reference image $I_{2 k-1}$ and the test image $I_{2 k}$ are compared, and zero transformations should be obtained in the other cases. Taking this fact into account, in each matching experiment only the affine transformation with the highest priority is considered. The other obtained transformations are not analyzed. Thus, TP (True Positive) and FP (False Positive) rates are estimated according to:

(i) TP rate is computed as the percent of correct best-priority transformations (just one per image can be correct).

(ii) FP rate is computed as the percent of incorrect best-priority transformations (just one incorrect transformation per image is added).

In the experiments five main parameters were analyzed: matches_max ratio, $l x, n x$, $d x \_$Bin and $d y \_B i n$. matches_max ratio (distance ratio between the best descriptor and the best second descriptor) controls the quality of the selected matches; $l x$ and $n x$ determinate the size of the descriptors; $d x \_$Bin and $d y \_B i n$ determinates the grid size of the Hough transform. In the first experiments it was found that when fixing the 
values of $l x$ to 8 and $n x$ to 4 high TP detections were obtained. From this starting point the other remaining parameters were further adjusted until a maximum detection rate of TP with a corresponding low FP rate were obtained. The final results are summarized in the table 1, where the best results are shown in bold.

It can be observed that the best obtained results correspond to the case when matches_max_ratio is set to 0.8 , and $d x \_B i n=d y \_B i n=0,10: \mathrm{TP}=87 \%$ and $\mathrm{FP}=1.7 \%$. Second best results are get with the combination matches_max ratio $=0.85$ and $d x \_B i n=d y \_B i n=0.10$ : TP=90\% and $\mathrm{FP}=7.6 \%$. It can also be noticed that the TP rate increases almost linearly as a function of the $d x_{-}$Bin and $d y_{-}$Bin parameters. Meanwhile, their corresponding FP rate results varies in a not linear way, showing that while these parameters are changed, they must affect some other parameters involved in the calculations. These effects can be observed in figure 1 .

Table 1. TP and FP rate results of our simulation having the algorithm tuning parameters $l x$ and $n x$ fixed to 8 and 4 respectively. matches_max ratio, $d x \_$Bin and $d y \_B i n$ are varied as shown.

\begin{tabular}{|c|c|c|c|c|c|c|}
\hline \multirow{2}{*}{ dx_Bin = dy_Bin } & \multicolumn{6}{|c|}{ matches_max_ratio } \\
\cline { 2 - 7 } & \multicolumn{2}{|c|}{$\mathbf{0 . 8 0}$} & \multicolumn{2}{c|}{$\mathbf{0 . 8 5}$} & \multicolumn{2}{c|}{ 0.90 } \\
\cline { 2 - 7 } & TP(\%) & FP(\%) & TP (\%) & FP (\%) & TP(\%) & FP(\%) \\
\hline 0.40 & 75 & 5.1 & 77 & 12.2 & 66 & 24.3 \\
0.35 & 82 & 5.4 & 78 & 12.7 & 75 & 25.6 \\
0.30 & 81 & 5.5 & 79 & 13.1 & 76 & 27.2 \\
0.25 & 82 & 5.2 & 82 & 13.7 & 76 & 28.1 \\
0.20 & 82 & 4.9 & 82 & 13.4 & 80 & 28.5 \\
0.15 & 87 & 3.4 & 88 & 11.6 & 80 & 28.4 \\
0.10 & $\mathbf{8 7}$ & $\mathbf{1 . 7}$ & $\mathbf{9 0}$ & $\mathbf{7 . 6}$ & 82 & 23.8 \\
\hline
\end{tabular}

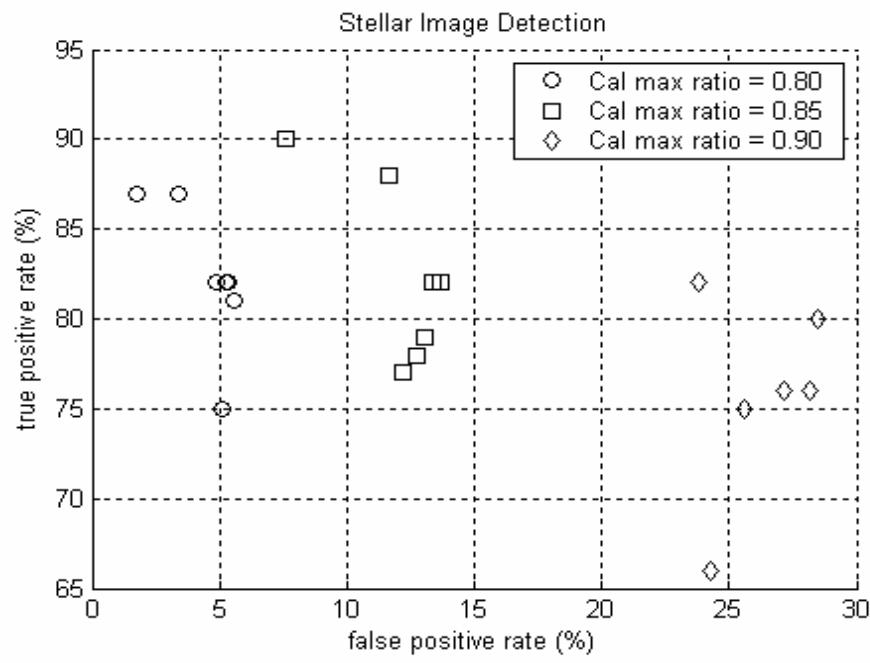

Fig. 1. TP ratio plotted against FP ratio according to the values of Table 1 


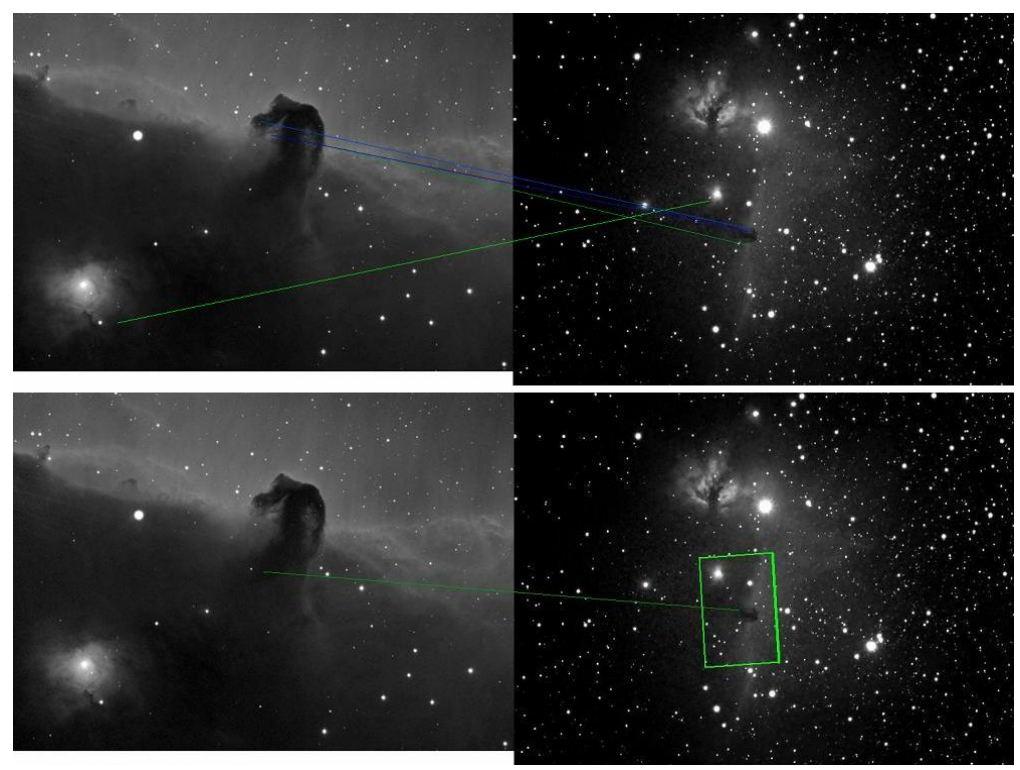

Fig. 2. A True Positive match example of the used algorithm applied on the "Horse nebula"
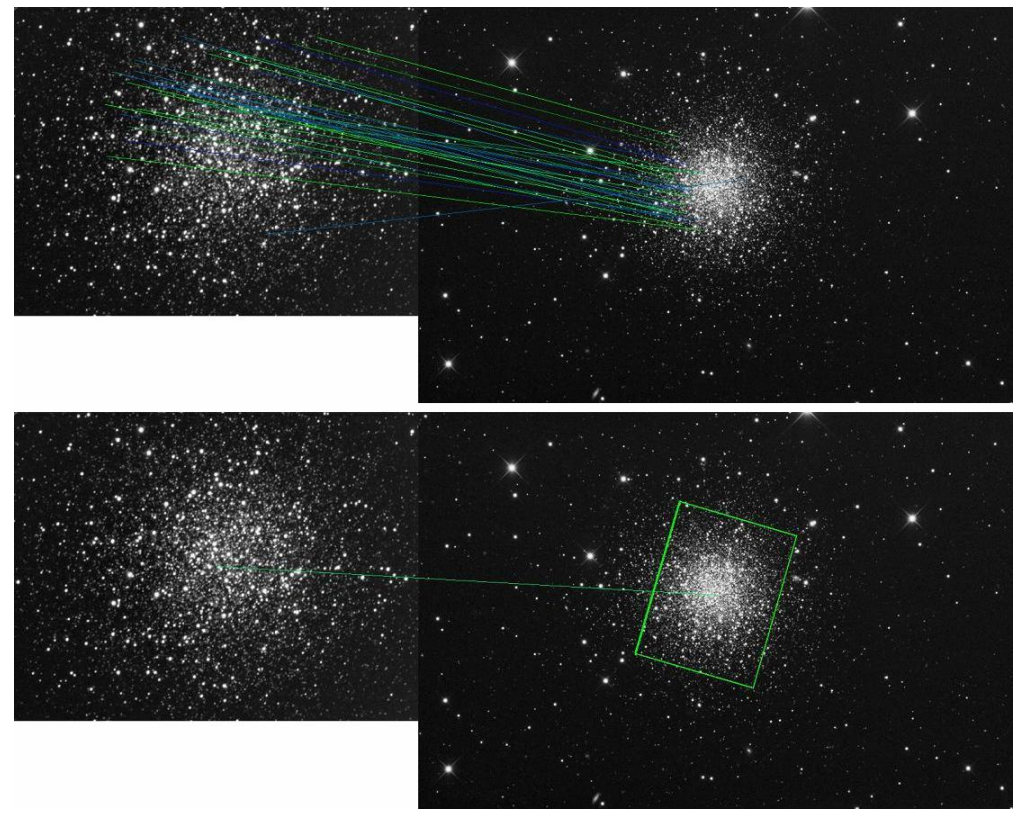

Fig. 3. TP match example obtained on the NGC_2808 Star Cluster 

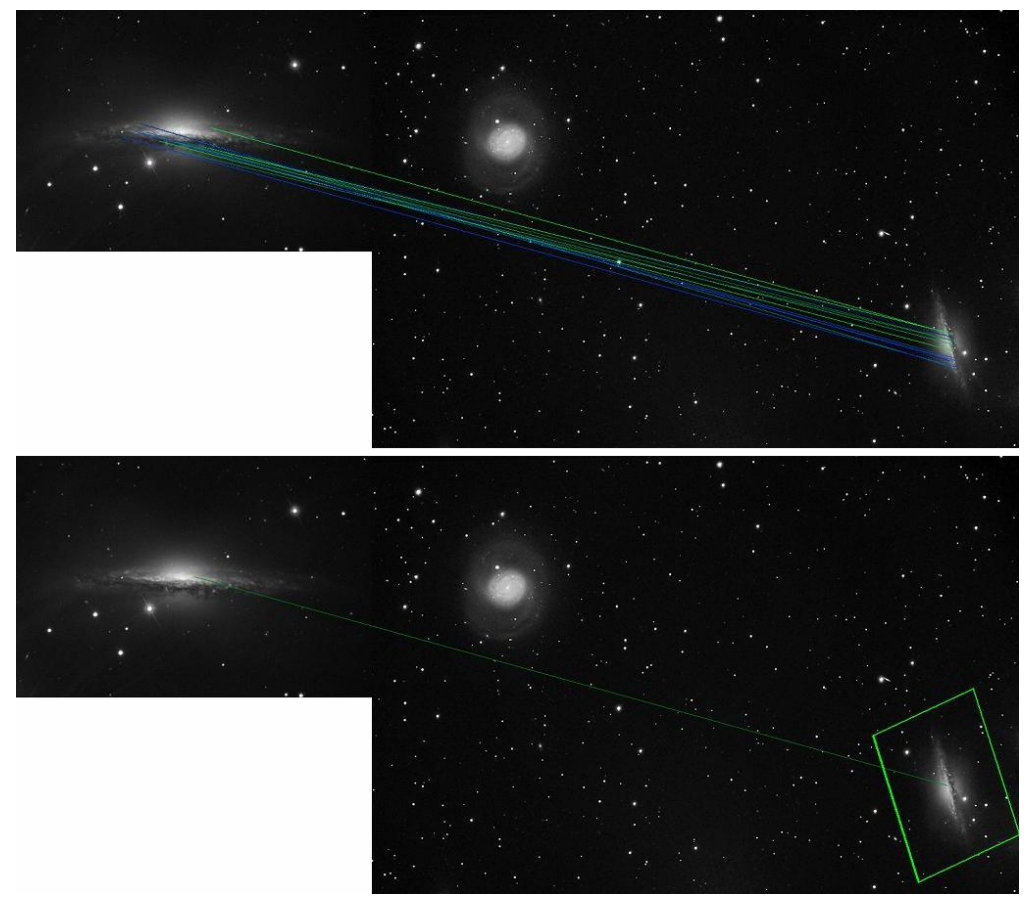

Fig. 4. NGC_1055 Galaxy TP matching result

Figure 2-4 show some exemplar results of how the proposed matching approach select the most similar matches (green and blue lines), allowing that before the total area of the reference image of interest is projected as a box over the test image.

\section{Conclusions}

In this paper was proposed a robust SIFT-based wide baseline matching approach for stellar images. The proposed methodology is validated using a stellar image database composed by 100 pair of galaxies, nebulas and star cluster images, which were specially selected from databases corresponding to different observatories around the world. Each reference image shows a different, single stellar object. The same object appears in the corresponding test image, which can contains variations in position and view angle, as well as size, resolution and camera parameters. In this database a true positive rate of $87 \%$ with a false positive rate of $1.7 \%$ is obtained, which is an excellent result due to the complexity of the matching problem.

From these good results it can be concluded that the methodology is a very useful pattern detection algorithm that can be applied in several other fields of astronomical image processing, where matching techniques for astronomical images are required. Such applications are for example stellar photometry, telescope tracking and pointing 
programs, robot (automated) telescopes, monitoring techniques, pattern recognition in data intensive astronomy, and in searching/matching CCD images to a stellar catalogues (see for example the astrometry engine project [15]).

\section{References}

1. Lowe, D.: Object Recognition from Local Scale-invariant Features. In: 7th IEEE International Conference on Computer Vision, pp. 1150-1157. IEEE Computer Society Press, California (1999)

2. Lowe, D.: Distinctive Image Features from Scale-Invariant Keypoints. International Journal of Computer Vision 60, 91-110 (2004)

3. Scovanner, P., Ali, S., Shah, M.: A 3-dimensional Sift Descriptor and its Application to Action Recognition. In: 15th International Conference on Multimedia, pp. 357-360. ACM Press, New York (2007)

4. Se, S., Lowe, D.G., Little, J.: Vision-based Mobile Robot Localization and Mapping Using Scale-invariant Features. In: IEEE International Conference on Robotics and Automation (ICRA), pp. 2051-2058. IEEE Press, New Jersey (2001)

5. Jo, Y.-G., Lee, J.-Y., Kang, H.: Segmentation Tracking and Recognition Based on Foreground-Background Absolute Features, Simplified SIFT, and Particle Filters. In: IEEE Congress on Evolutionary Computation, pp. 1279-1284. IEEE Press, New York (2006)

6. Harris, C., Stephens, M.: A combined corner and edge detector. In: 4th Alvey Vision Conference, pp. 147-151. Manchester, UK (1998)

7. Lowe, D.: Local feature view clustering for $3 D$ object recognition. In: IEEE Conference on Computer Vision and Pattern Recognition, pp. 682-688. IEEE Press, Los Alamitos (2001)

8. Ferrari, V., Tuytelaars, T., Van Gool, L.: Simultaneous Object Recognition and Segmentation by Image Exploration. In: Pajdla, T., Matas, J(G.) (eds.) ECCV 2004. LNCS, vol. 3021, pp. 40-54. Springer, Heidelberg (2004)

9. Mikolajczyk, K., Schmid, C.: Scale \& Affine Invariant Interest Point Detectors. International Journal of Computer Vision 60, 63-96 (2004)

10. Loncomilla, P., Ruiz-del-Solar, J.: A Fast Probabilistic Model for Hypothesis Rejection in SIFT-Based Object Recognition. In: Martínez-Trinidad, J.F., Carrasco Ochoa, J.A., Kittler, J. (eds.) CIARP 2006. LNCS, vol. 4225, pp. 696-705. Springer, Heidelberg (2006)

11. Loncomilla, P., Ruiz-del-Solar, J.: Gaze Direction Determination of Opponents and Teammates in Robot Soccer. In: Bredenfeld, A., Jacoff, A., Noda, I., Takahashi, Y. (eds.) RoboCup 2005. LNCS (LNAI), vol. 4020, pp. 230-242. Springer, Heidelberg (2006)

12. Loncomilla, P., Ruiz-del-Solar, J.: Improving SIFT-based Object Recognition for Robot Applications. In: Roli, F., Vitulano, S. (eds.) ICIAP 2005. LNCS, vol. 3617, pp. 1084 1092. Springer, Heidelberg (2005)

13. Ruiz-Del-Solar, J., Loncomilla, P., Devia, C.: A New Approach for Fingerprint Verification based on Wide Baseline Matching using Local Interest Points and Descriptors. In: Mery, D., Rueda, L. (eds.) PSIVT 2007. LNCS, vol. 4872, pp. 586-599. Springer, Heidelberg (2007)

14. Schmid, C., Mohr, R.: Local grayvalue invariants for image retrieval. In: IEEE Transactions Pattern Analysis and Machine Intelligence, pp. 530-534. IEEE Computer Society Press, California (1997)

15. Astrometry Engine Project Website, http://astrometry.net 